\title{
Mid-term trends and recent birth-cohort-dependent changes in incidence rates of cutaneous malignant melanoma in Italy
}

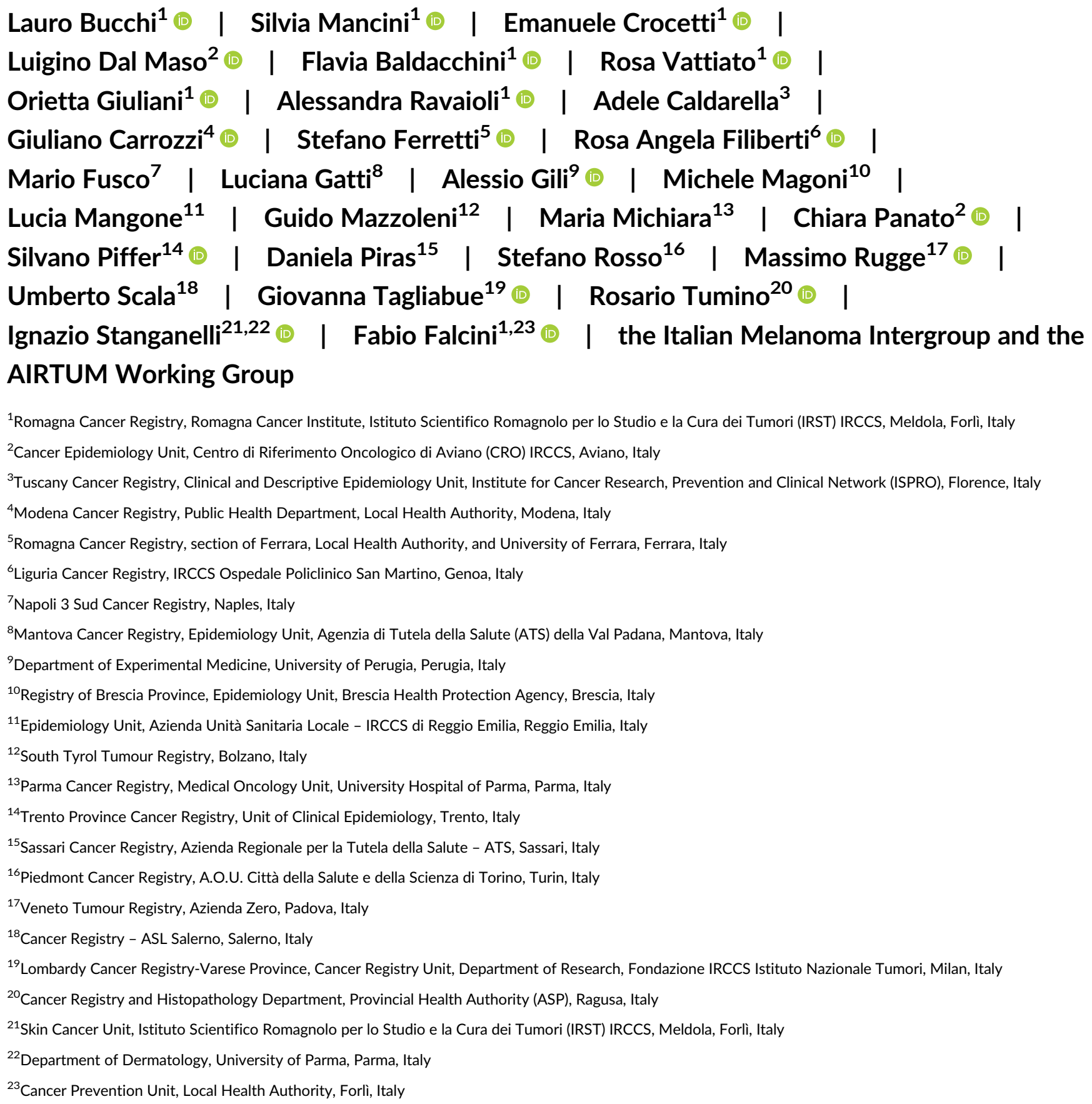




\section{Correspondence}

Silvia Mancini, Romagna Cancer Registry, Romagna Cancer Institute (Istituto Scientifico Romagnolo per lo Studio e la Cura dei Tumori (IRST) IRCCS), 47014 Meldola, Forlì, Italy,

Email: silvia.mancini@irst.emr.it

Funding information

Italian Melanoma Intergroup; Italian Ministry of Health

\section{Abstract}

In Oceania, North America and north-western Europe, after decades of increase, cutaneous malignant melanoma (CMM) rates began to stabilise or decline before 2000. Anecdotal evidence suggests that the reversal of the incidence trend is extending to southern Europe. To obtain a formal confirmation, this nationwide study from Italy investigated the incidence trends by birth cohort. Twenty-one local cancer registries covering a population of 15814455 provided incidence data for primary CMM registered between 1994 and 2013. Trends in age-standardised rates were analysed using joinpoint regression models and age-period-cohort models. Age-standardised incidence showed a consistent increase throughout the period (estimated annual percent change, 3.6 [95\% confidence interval, 3.2-4.0] among men and 2.5 [2.0-3.1] among women). This pattern was confirmed by a sensitivity analysis with removal of low-risk populations of southern Italy. The rates, however, showed a stabilisation or a decrease in men and women aged below 35. Using the cohort of 1949-the median cohort with respect to the number of cases for both genders-as a reference, the incidence rate ratio increased for successive cohorts born until 1973 (women) and 1975 (men), and subsequently tended to decline. For the most recent cohorts in both genders, the risk of disease returned to the level of the cohort of 1949 . The changes observed in the latest generations can be interpreted as the earliest manifestations of a birth-cohort-dependent incidence decrease. Our study adds to previous data indicating that the reversal of the long-term upward incidence trend of CMM is extending to southern Europe.

\section{KEYWORDS}

age-period-cohort modelling, birth cohort, cutaneous malignant melanoma, incidence, time trend

\section{1 | INTRODUCTION}

Since approximately the middle of the 20th century, but with remarkable temporal differences, the incidence of cutaneous malignant melanoma (CMM) has increased for decades in the greater part of Caucasian populations worldwide. ${ }^{1-9}$ This trend has been caused by a deep change in attitudes towards sun bathing, from a sun-avoidance to a sun-seeking behaviour, ${ }^{10}$ with intermittent exposure to the ultraviolet radiation, ${ }^{11}$ coupled with increased use of artificial ultraviolet radiation sources.

In many populations, however, a slowing of the upward incidence trend has been observed in the most recent decades, generally followed by a stabilisation and a decline. ${ }^{1,6,10,12-15}$ In Australia, for example, the rates increased until around 1990. The risk of disease peaked for men born before 1930, stabilised in the birth cohorts of 1930 to 1950 , and fell in subsequent ones. Women showed similar changes but about 5 years earlier. ${ }^{13,14,16,17}$ In the United States, the risk stabilised before the turn of the century for women younger than 60 years and men younger than 50 year. ${ }^{12}$ In the following decade,

\section{What's new?}

After increasing for many years, malignant melanoma rates began to stabilise or decline in many regions before 2000 . Is this also true for southern Europe? In the current study, the authors found that melanoma rates have also began to decline in Italy. The reversal of the long-term, worldwide increase in melanoma risk seen in other Caucasian populations thus appears to extend to southern Europe. These results indicate that primary prevention strategies have been effective in modifying sun-exposure habits in this region, and should continue to be reinforced via public health messaging.

significant decreases were seen among young adults. ${ }^{18}$ In detail, the risk increased for successive cohorts of non-Hispanic whites born between 1921 and 1981 before starting to decrease. ${ }^{19}$

As regards Europe, the incidence increase has been generalised in the last 50 and more years. ${ }^{6}$ The trend has started in northern Europe 
and has spread to the south. ${ }^{6,20}$ In north-western European countries, the increase has been strong until the 1980s, after which the rates have stabilised or declined starting from the cohorts of 1930 to $1940 .^{6}$

So far, conversely, no stabilisation or decrease in incidence has taken place in southern Europe, that is, in central Mediterranean ${ }^{21-23}$ as well as in south-western ${ }^{24-26}$ and south-eastern Europe. ${ }^{27}$ This part of the continent has experienced comparatively moderate increases in incidence, but this trend has been predicted to persist in the foreseeable future. This is the case, among others, for Spain ${ }^{25,26}$ and Italy. ${ }^{21-23}$

Recently, however, small/mid-sized regional studies from these two countries have provided anecdotal evidence that the risk of CMM has begun to decrease starting with the latest cohorts. A decrease in incidence has been reported from Catalonia in the age range of 25 to 29 years in the first decade of this century. ${ }^{28} \mathrm{~A}$ study from northern Italy has revealed a slight decrease among women born after 1961 and an attenuation of the increasing trend in the latest cohorts of men. ${ }^{29}$ These observations could herald that the reversal of the long-term incidence trend of CMM is extending to southern Europe. This provided the rationale for this nationwide study from Italy, whose objective was to obtain a formal confirmation by investigating the incidence trends by birth cohort.

\section{2 | MATERIALS AND METHODS}

\section{1 | Data}

The data for the study were derived from the database of the Italian Association of Cancer Registries (AIRTUM). ${ }^{30,31}$ Thirty-eight registries authorised their records to be accessed. When the study was undertaken (October 2019), their common dataset covered the years 1976 to 2016. The initial and final years of this time span, however, were excluded because the number of operating registries (11 in 1976-1993 and 8 in 2014-2016) was considered insufficient. In addition, for the core period 1994 to 2013, we selected the 21 registries that had been operating for $\geq 15$ years. Data for primary invasive $\mathrm{CMM}$ were extracted using the International Statistical Classification of Diseases and Related Health Problems, 10th revision, codes C43.0 to C43.9. ${ }^{32}$

Table S1 gives the years of registration of the eligible registries, the populations covered, the number of CMM cases contributed to the study and the indices of data quality. In particular, the proportion of "death certificate only" cases varied between $0.0 \%$ and $0.6 \%$ among men and between $0.0 \%$ and $0.7 \%$ among women. Table S2 gives the 5 -year age distribution of the study population and of CMM cases. On 31 December 2003, the mid-point in the study period, the total population coverage

TAB LE 1 Age-period-cohort models for trends in incidence of cutaneous malignant melanoma in Italy from 1994 to 2013 , by gender (population aged $15-84$ years)

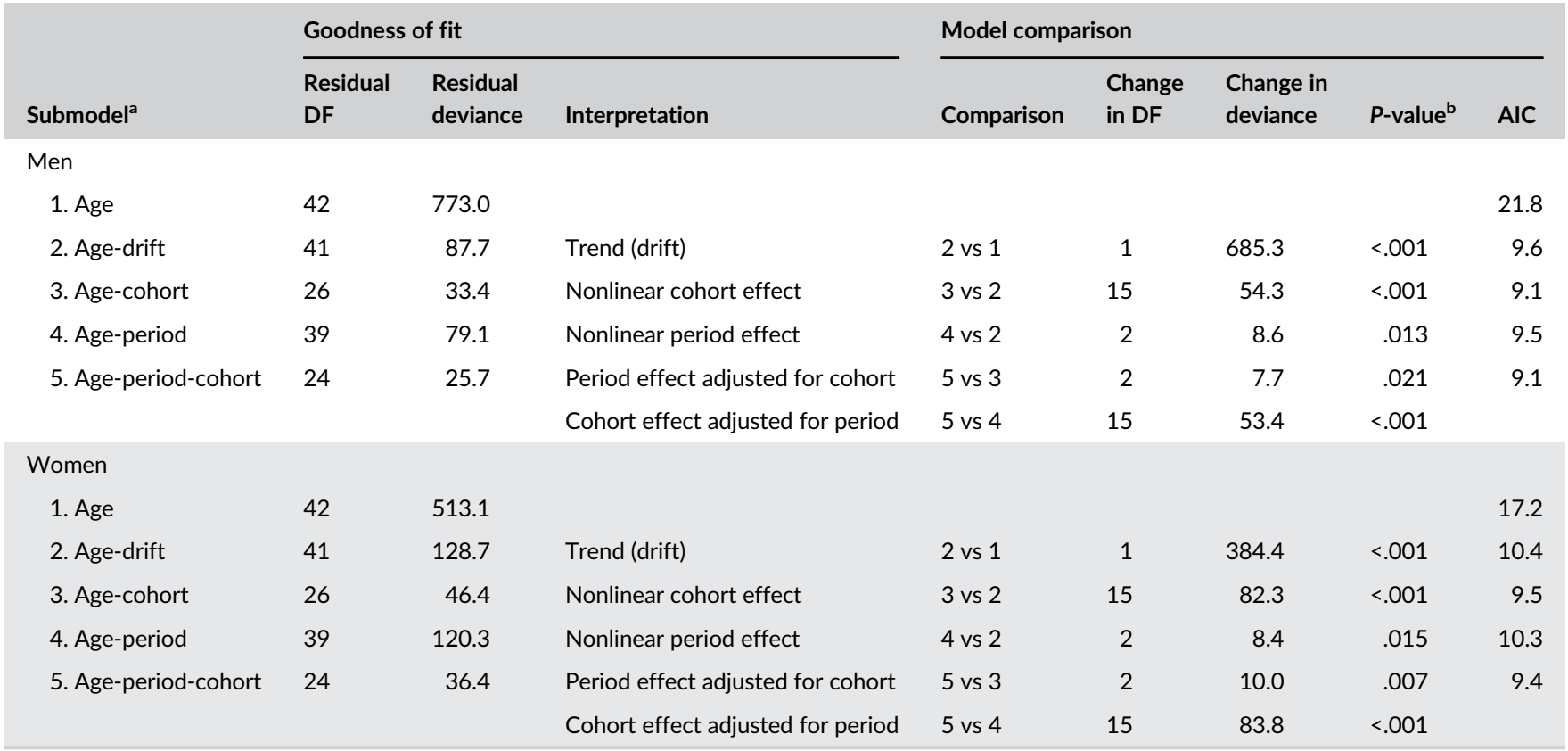

Abbreviations: AIC, Akaike information criterion; DF, degrees of freedom.

${ }^{a}$ Five submodels were derived, namely: age, age-drift, age-cohort, age-period and the full age-period-cohort model. The drift term is a linear temporal variation of rates that cannot be attributed to either the period effect or the cohort effect. The model goodness-of-fit was tested using residual deviance statistics. The age, period and birth cohort effects were derived from pairwise comparisons of the appropriate submodels. The statistical significance of the pairwise comparisons was tested by comparing the difference in residual deviance and degrees of freedom using the likelihood ratio test. The models 3 and 4 could not be compared directly in this way nor was it possible to perform (and it was not possible to construct) a formal test of whether the agecohort model was significantly better than the age-period model. In both genders, the full age-period-cohort model provided the best fit to the data, as confirmed by the observation that the full age-period-cohort model minimised the AIC both for men and women.

bikelihood ratio test. 
was 15814455 (27.5\% of the Italian population). Between 1994 and 2013 , a total of 45264 CMM cases were registered.

\section{2 | Statistical methods}

The data were first analysed descriptively. The trends in agestandardised (2013 European standard population) incidence rates were assessed using the joinpoint regression analysis. All registries were included. The estimated annual percentage change (EAPC) was calculated with generalised linear models assuming a Poisson distribution.

Given the small number and size of registries of southern Italy (Table S1) as well as their lower rates of CMM (Results section), we performed a sensitivity analysis to explore the hypothesis that they could cover epidemiologically selected populations with a different incidence trend from that of the other registries. All joinpoint regression models were re-run after exclusion of the registries of southern Italy. On 31 December 2003 (the mid-point in the study period), they accounted for a population of 1155890 men (15.1\% of the total number of men in the study population) and 1207631 women (14.8\%). They contributed to the study 1618 and, respectively, 1738 CMM cases.

Subsequently, we performed an age-period-cohort analysis ${ }^{33,34}$ of the incidence trends. Based on the results of the sensitivity analysis, all registries were included. Due to the instability of rates, people aged $<15$ years and $>84$ years were excluded. They were comprised of 1185914 men and 1287417 women with 873 and 1321 CMM cases, respectively. This left 6467442 men and 6873682 women, with 21579 and 21491 CMM cases, available for analysis. The incidence data were tabulated into fourteen 5-year age groups, four 5 -year periods and seventeen overlapping 10-year birth cohorts. ${ }^{35}$ The cohorts were obtained by subtracting the mid-point of the 5-year age group (from 15-19 years to 80-84 years) from the corresponding mid-point of the 5 -year period. The cohorts were identified by their mid-year of birth. The age-specific rates were plotted against the cohort and the period.

Finally, we fitted five Poisson regression models according to the model-building approach. ${ }^{34}$ The model goodness-of-fit was assessed using residual deviance statistics. Models were compared using conditional likelihood ratio tests between hierarchically nested models and the Akaike information criterion. ${ }^{36}$ Further details of model fitting are provided in Table 1.

To overcome the problem of nonidentifiability, we constrained the linear component of the period effect to have zero slope, thus assuming that the linear changes in incidence of disease were due to cohort-related factors. ${ }^{10}$ The linear trend (drift) was allocated to the cohort. The median cohort with respect to the number of cases, that
Men ASR, 20.1

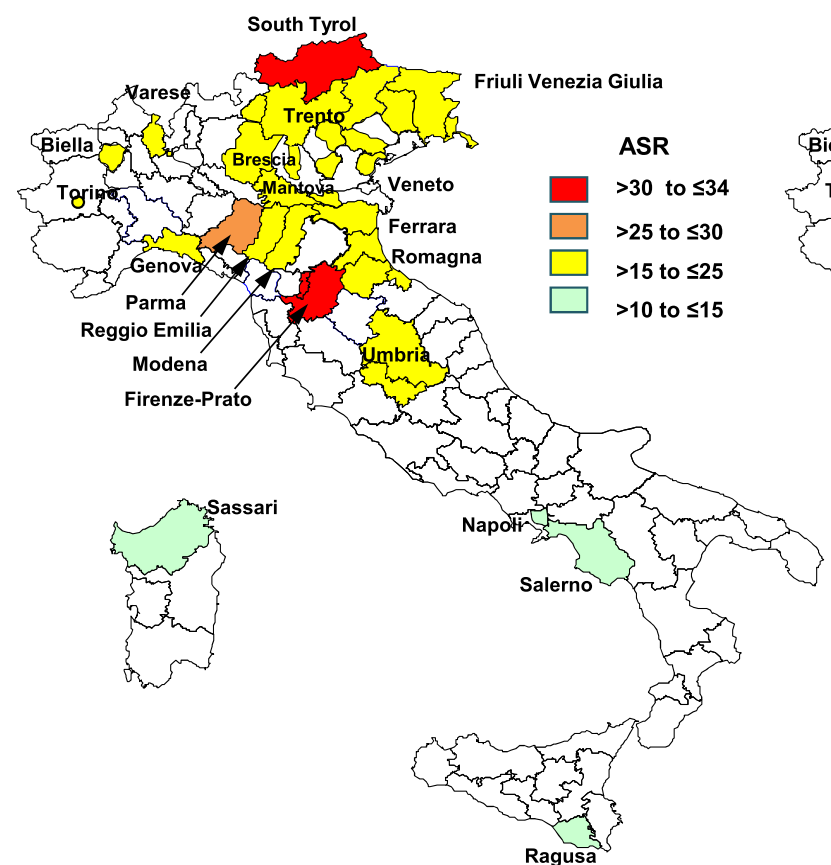

Women ASR, 16.4

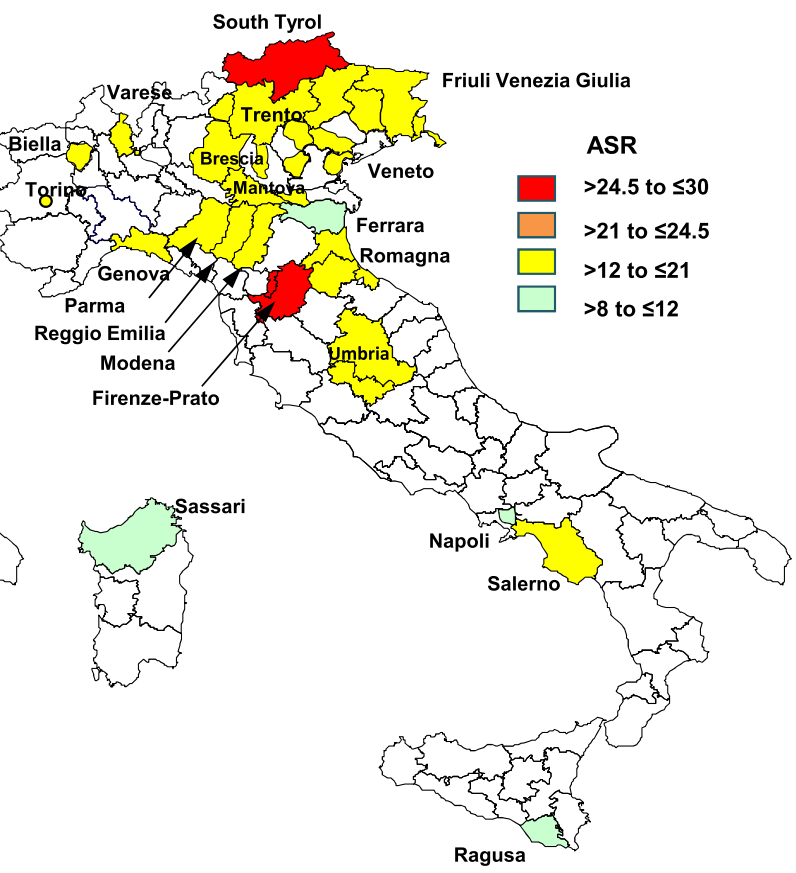

, >1.5 times the Italian average ASR;

, $>1.25$ to $\leq 1.50$;

$>0.75$ to $\leq 1.25$

$>0.5$ to $\leq 0.75$;

FIGURE 1 Absolute and categorised average annual age-standardised (2013 European standard population) incidence rate (per 100 000 ) of cutaneous malignant melanoma in Italy from 2009 to 2013, that is, the most recent 5-year period of data contribution, by gender and cancer registration area. ASR indicates age-standardised rate. ASRs are categorised according to their ratio to the Italian average ASR. This type of grouping illustrates the pronounced incidence gradient from northern-central Italy to southern Italy 

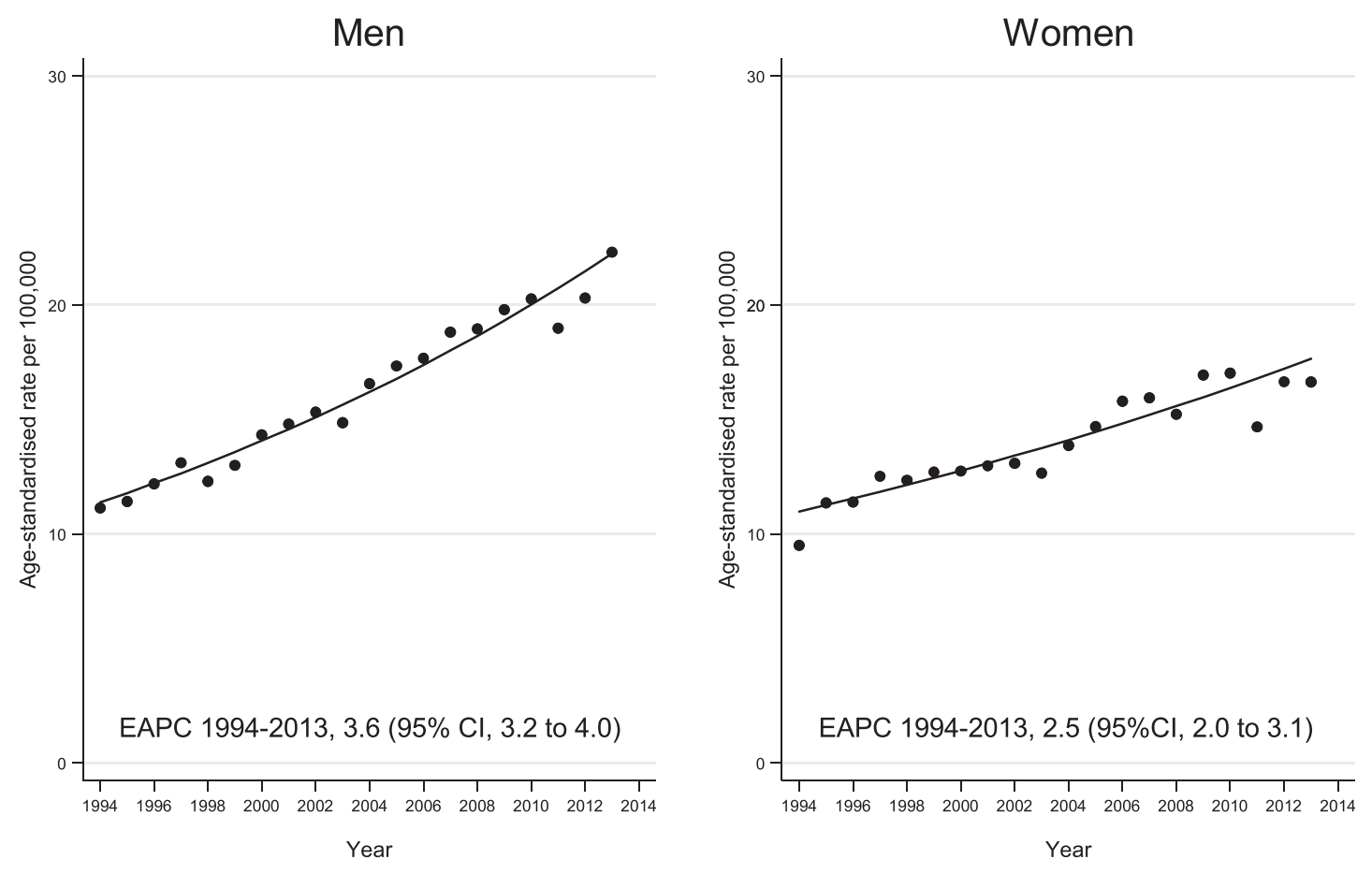

\section{- Observed - Modelled}

FIGURE 2 Observed and joinpoint-modelled annual age-standardised (2013 European standard population) incidence rate (per 100 000 ) of cutaneous malignant melanoma in Italy from 1994 to 2013, by gender. The trends in incidence rates were analysed using the joinpoint regression, which enabled to detect the point in time when a change in direction or magnitude of the trend occurred (if any). EAPC indicates the estimated annual percentage change in incidence rates for each time segment. $95 \% \mathrm{Cl}$ indicates $95 \%$ confidence interval. Age-standardised incidence showed a regular and significant increasing trend

TABLE 2 Sensitivity analysis of the estimated annual percent change in age-standardised (2013 European standard population) incidence rate (per 100 000) of cutaneous malignant melanoma in Italy from 1994 to 2013, by gender and age (population aged 15 years or older)

\begin{tabular}{|c|c|c|c|c|c|}
\hline $\begin{array}{l}\text { Cancer registries removed from the } \\
\text { joinpoint regression models }\end{array}$ & Age (years) & \multicolumn{2}{|l|}{ Men } & \multicolumn{2}{|l|}{ Women } \\
\hline \multirow[t]{4}{*}{ None } & $15-24$ & $1994-2013$ & $2.0(-0.5$ to 4.6$)$ & $1994-2013$ & $1.0(-1.0$ to 2.9$)$ \\
\hline & $25-44$ & $1994-2013$ & 3.4 (2.7 to 4.1$)$ & $1994-2013$ & 3.7 (2.9 to 4.5 ) \\
\hline & $45-64$ & $1994-2013$ & 2.9 (2.3 to 3.6$)$ & $1994-2013$ & 2.8 (2.2 to 3.4$)$ \\
\hline & $\geq 65$ & $1994-2013$ & 4.2 (3.8 to 4.6 ) & $1994-2013$ & 1.5 (1.0 to 2.0 ) \\
\hline \multirow[t]{4}{*}{ Registries of southern Italy } & $15-24$ & $1994-2013$ & $2.5(-0.4$ to 5.5$)$ & $1994-2013$ & 1.2 (-1.1 to 3.6$)$ \\
\hline & $25-44$ & $1994-2013$ & 3.4 (2.6 to 4.2 ) & $1994-2009$ & $4.4(3.2$ to -5.6$)$ \\
\hline & & & & $2009-2013$ & $-3.5(-11.2$ to 5.0$)$ \\
\hline & $45-64$ & $1994-2013$ & 3.1 (2.4 to 3.8 ) & $1994-2013$ & 2.8 (2.1 to 3.5 ) \\
\hline
\end{tabular}

Abbreviations: $\mathrm{Cl}$, confidence interval; EAPC, estimated annual percent change.

of 1949 for both genders, was used as a reference. ${ }^{10}$ The cohort effect was interpreted as an incidence rate ratio (IRR). With the inclusion of a slope for the cohort effect, the age effect became interpretable as age-specific rate for the cohort of 1949 after adjustment for the period effect. The period effect, set to 1 on average with zero slope, can be interpreted as the period-related IRR after 
adjustment for age and cohort. Age, period and cohort effect estimates were modelled using restricted cubic spline functions. Pointwise confidence intervals ( $\mathrm{Cls}$ ) were computed.

The data analysis and the presentation of model estimates were made using the STATA package version 15.1 (Stata Corporation, College Station, Texas) and the APCfit STATA command. ${ }^{37}$

\section{3 | RESULTS}

Table S3 shows, for each of the eligible cancer registries and the three broad geographic areas of Italy, the incidence rate of CMM in the earliest and in the most recent 5-year period of data contribution, and the EAPC over the entire period of data contribution. There was a large difference between the pool of registries of northern-central Italy and those of southern Italy, with approximately 2-fold higher age-standardised rates for the former both in 1994 to 1998 and in 2009 to 2013.

The maps in Figure 1 are based on the most recent 5 -year period of data contribution by each registry. Age-standardised rates are categorised according to their ratio to the average Italian age-standardised rate. This categorisation provides an accurate representation of the marked north-south geographic gradient in incidence of CMM across the country.

\subsection{Joinpoint regression analysis}

Figure 2 shows the curve of annual incidence rate by gender. There was a consistent and significant upward trend both among men and women, with an EAPC of 3.6 and 2.5, respectively.

The case series included 898 (2.0\%) CMM cases aged 15-24 years, 10589 (23.4\%) aged 25-44 years, 16178 (35.8\%) aged $45-64$ years and 17535 (38.8\%) aged $\geq 65$ years. The results of the joinpoint regression analysis in these broad age groups confirmed a generalised increase both among men and women, although the EAPC was smaller and no longer significant for women aged 15-24 years (upper section of Table 2). In sensitivity analysis, the removal of the registries of southern Italy from the models (bottom section of Table 2) confirmed this pattern of incidence.

\subsection{Age-period-cohort analysis}

All registries were included in the age-period-cohort analysis. Figure 3 shows the 5 -year age-specific incidence rate by 5 -year period of diagnosis (Figure 3A,B) and 10-year birth cohort (Figure 3C,D). Uniformly increasing trends were found across all age groups but with a recent stabilisation in people of both genders aged below 35 years. Incidence curves by age group and birth cohort on a semi-logarithmic scale had different slopes
(A)

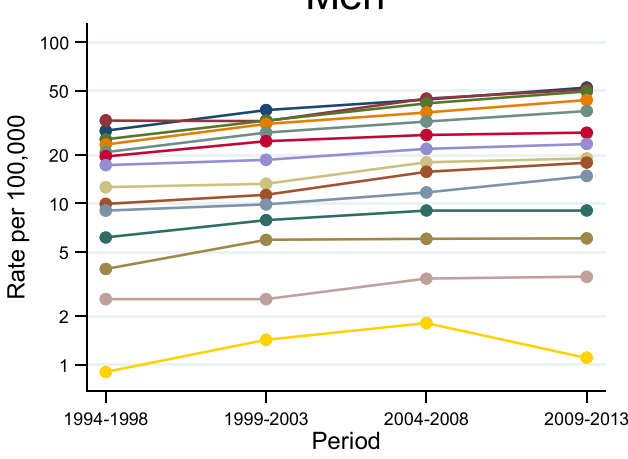

(C)

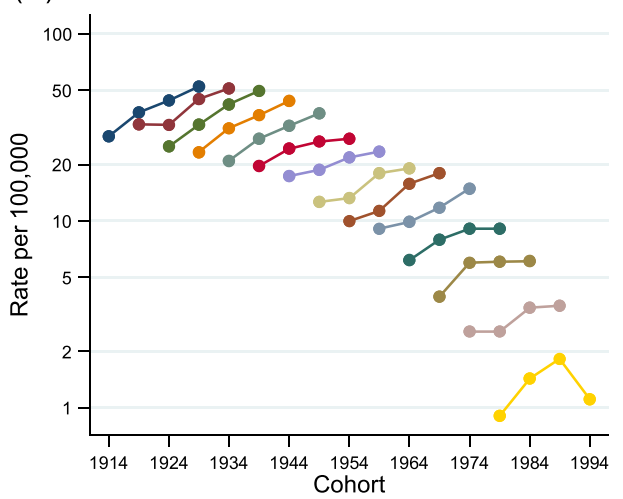

(B)

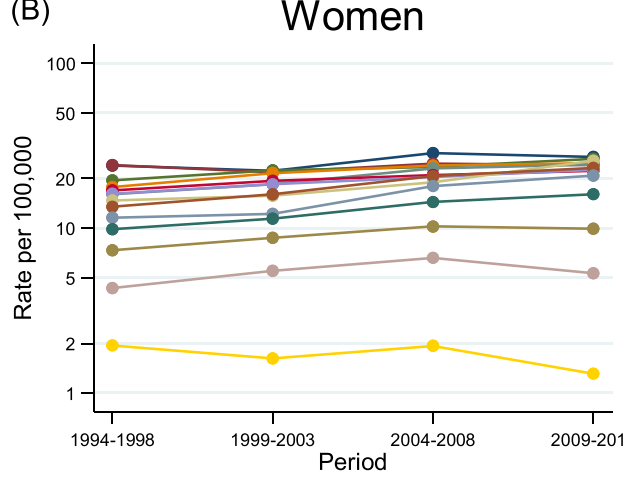

(D)

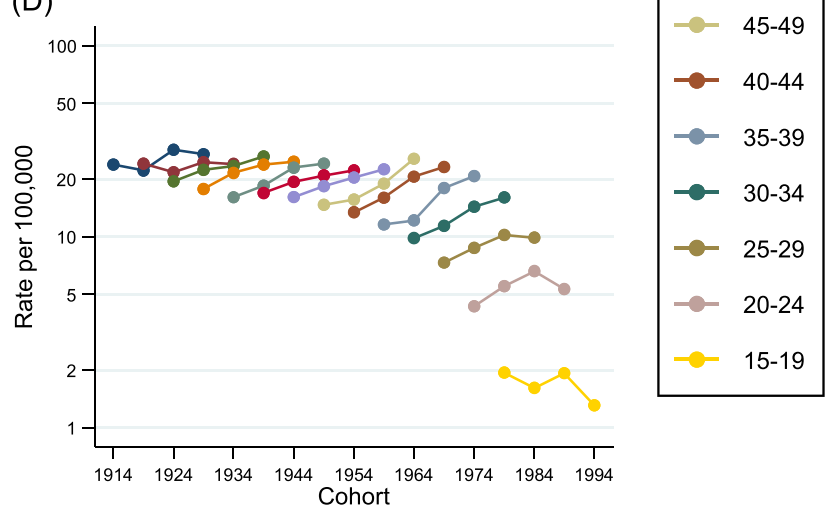

FIGURE 3 Five-year age-specific incidence rate (per 100 000) of cutaneous malignant melanoma in Italy from 1994 to 2013 on a semilogarithmic scale, by gender, 5-year period of diagnosis (Figure 3A,B) and 10-year birth cohort (Figure 3C,D) (population aged 15-84 years). The rates for each age group are joined by lines and are plotted against the mid-year of birth. For the younger age groups of both genders, a stabilisation and a small decrease in age-specific incidence rates were observed 

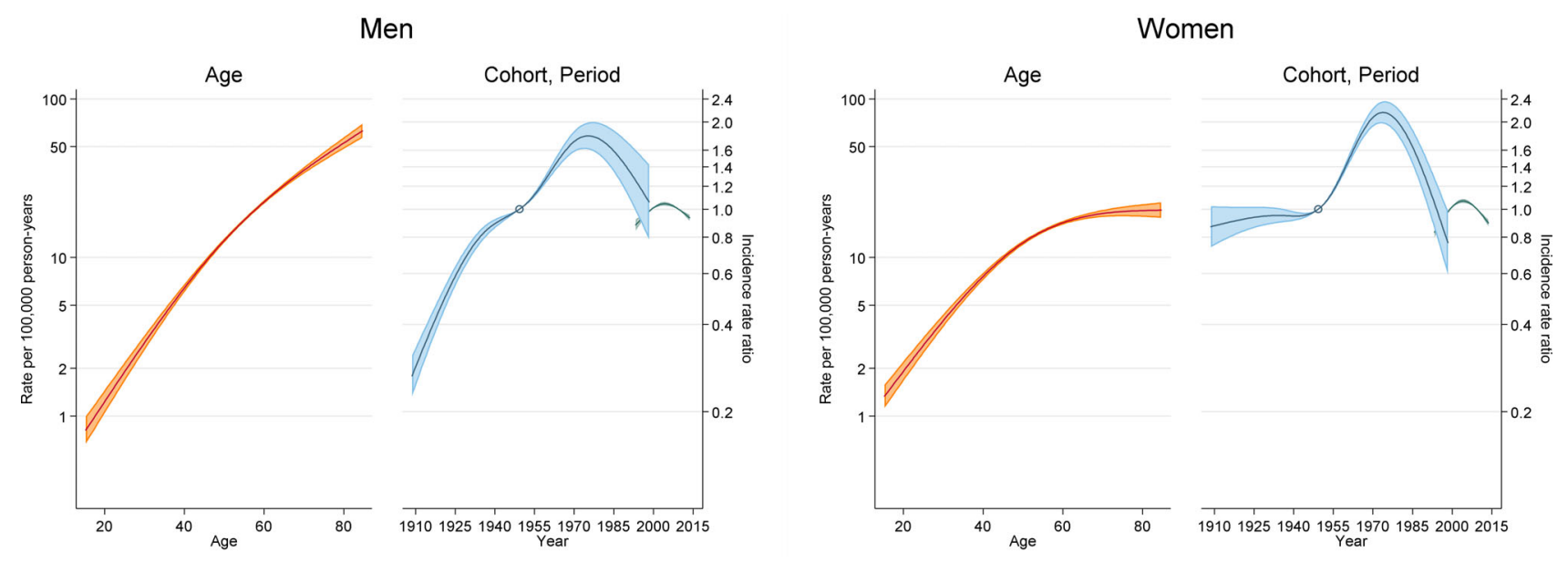

FIGURE 4 Age, cohort and period effects on incidence of cutaneous malignant melanoma in Italy from 1994 to 2013, by gender (population aged 15-84 years). One internal knot for age and period and three internal knots for the cohort variable were used. Using the cohort of 1949the median cohort with respect to the number of cases for both genders-the incidence rate ratio increased for successive cohorts of women born until 1973 and of men born until 1975. Subsequently, the risk tended to decline

(Figure 3C,D). This is suggestive of a cohort effect. Incidence rate by cohort increased consistently in all age groups with two exceptions: the oldest women, aged 70 years and above, for whom the risk of disease was substantially stable over the study period; and, more important, the younger age groups of both genders, in whom a stabilisation and a small decrease in age-specific incidence rates were observed.

In the age-period-cohort modelling analysis, the full model provided the best fit to the data (Table 1). The relative contribution of the estimated effects of age, period and cohort is illustrated in Figure 4. Using the cohort of 1949 as a reference in both genders, the IRR increased for successive cohorts of women born until 1973 and of men born until 1975. Subsequently, it tended to decline. For the most recent cohorts of both men and women, the risk of disease returned to the level of the cohort of 1949.

\section{4 | DISCUSSION}

The primary findings of our study are that (a) age-standardised incidence of CMM increased regularly between 1994 and 2013 for both genders, and (b) the rates rose for all cohorts born until the mid-1970s and decreased for subsequent cohorts. This recent change can be interpreted as the earliest manifestation of a birth-cohort-dependent decrease in the risk of CMM in southern Europe. Thus, our study suggests that the results of the local, and smaller, studies from Catalo$\mathrm{nia}^{28}$ and, in particular, from northern Italy ${ }^{29}$ were not due to chance. Two recent mortality studies from Spain have yielded further supporting data. ${ }^{38,39}$

In addition, our findings provide the confirmation of the spatialtemporal pattern of change in the incidence of CMM in Europe. The incidence increase has diffused from the north to the south in an almost regular manner. ${ }^{6}$ As a stabilisation and even a decrease have already started to occur in northern Europe, the data in our study confirm that the reversal of the trend moves in the same geographic direction. ${ }^{10}$
A secondary but important observation is the stability of incidence among women aged 70 years and above. This suggests that the earliest cohorts of women, born in the 1920s, have not experienced major changes in sun exposure habits during their lifetime. Among men, conversely, the rate increased regularly for all cohorts including the earliest ones. These mostly included workers labouring outdoors, in particular agricultural and construction workers. Although outdoor work is more closely related with keratinocyte skin cancers, it conveys an increased risk of $\mathrm{CMM}^{40}$

The most obvious interpretation of these findings is that sun protection promotions have convinced the last generations of Italians to adopt safe sun exposure habits. ${ }^{15}$ It is worth noting that, elsewhere, the most pronounced decrease in the risk of CMM has been observed in those cohorts who were targeted by sun protection messages from the childhood. ${ }^{17}$ A different, but not alternative, hypothesis is that the information campaigns have had an impact on sunbathing habits in Italy when the increase in incidence of CMM has caused a sufficient level of public alarm. ${ }^{29}$ Given the delayed onset of the CMM epidemic $^{20}$ and the lower baseline rates, this has occurred later in Italy compared to other western countries.

Theoretically, an increased public awareness of the harms of exposure to ultraviolet radiation could not be the only cause of the reduction in sun exposure in the most recent cohorts. Other potential (and not necessarily alternative) explanations could include an increase in indoor leisure activities (eg, playing video games and using computers) ${ }^{17}$ and the immigration of individuals with darker complexion and at lower risk of disease. ${ }^{28}$ However, the percentage of leisure time spent using computers and the internet by the Italian population aged $\geq 15$ years, $6 \%$, is among the lowest in Europe, although the figure is 3-fold greater among people aged 15-24 years. Conversely, the percentage spent outdoors, $14 \%$, remains the highest.${ }^{41}$ Similarly, the immigration of individuals at lower risk of disease is likely to play a lesser role in Italy and other Mediterranean countries than in northern Europe, since the native Mediterranean-type populations have a more 
pigmented skin, that is, of Fitzpatrick phototype $\mathrm{IV},{ }^{42}$ than native populations in northern Europe. ${ }^{28}$

In fair-skinned populations, excessive sunbathing is the paramount risk factor for CMM and, consequently, the epidemiology of the disease does not change substantially throughout life. For this reason, the risk decrease being currently observed in the most recent birth cohorts in Italy is expected to persists as these cohorts age. If this prediction is confirmed, generations of men and women with increasingly lower risk of disease will replace generations at higher risk. By implication, agestandardised incidence rates will stabilise and then decline. ${ }^{20}$

It must be emphasised, however, that no declines in crude rates of $\mathrm{CMM}$ are anticipated for the next future in any western country, since the elderly will have an increasing representation in the population and will continue to have a high risk of disease. ${ }^{43}$ In Italy, the trends in age-standardised rates, too, will remain unfavourable for some decades, because of the time lag between changes in behaviour by the latest cohorts and their reflection in overall incidence.

So far, because of the incidence increase occurring in young adults, the number of life-years lost due to CMM death has been higher than that attributable to many other solid tumours. ${ }^{15}$ The future incidence trends and the underlying cohort effect, however, will be paralleled by an increase in patient age at diagnosis. ${ }^{20}$ Since the prognosis is currently worse for Italian patients above the middle age, ${ }^{44}$ it is likely that this age transition, which will be boosted by the demographic trend, will adversely affect survival rates. For the future, some have proposed that secondary preventive measures be increasingly targeted at older people. ${ }^{29,45}$

At its inception, a birth-cohort-dependent change in the risk of CMM involves a subset of the population that is small and youngaged, which limits the denominator and, respectively, the numerator of incidence rates. This means that a great statistical power is needed to demonstrate an initial decrease in incidence of CMM. From this, it follows that the major strength of the present study lies in its size.

Its major limitation is that cancer registration in Italy relies on a changing pool of registries covering-in the mid-point in the study period-one-fourth of the Italian population. Although we obtained data for a time span ranging from 1976 to 2016, we excluded the initial and final years because of the low number of operating registries. In particular, we truncated the study period at 2013. The years 2014-2016 would probably offer further proof of the ongoing incidence change. These problems arise from the fact that cancer registries have been introduced in Italy in a phased manner and, in the early years, they were concentrated in the north of the country. Only very recently has southern Italy been entirely covered. In general, the uneven distribution and the changing composition of the pool of registries have been dealt with by researchers in three ways: selecting a small group of historical registries, ${ }^{46}$ limiting the analysis to relatively short time periods with higher numbers of active institutions ${ }^{47}$ and performing a sensitivity analysis. ${ }^{48}$ In the present study, we employed both of the latter two approaches. In particular, sensitivity analysis suggested no significant heterogeneity in terms of incidence trends between the available registries of southern Italy and those of northern-central Italy.
Two methodological issues should be noted. First, our solution to deal with the problem of nonidentifiability was to assume that the linear changes in incidence of CMM resulted from cohort-related factors. This approach has been used in numerous studies on cancer incidence trends. ${ }^{10,49}$ Nonetheless, some degree of caution is needed in considering the results.

And second, studies on the incidence of CMM may be subject to biases. Improved disease reporting ${ }^{29,45}$ and increasing overdiagnosis ${ }^{22}$ over time may have contributed to the upward trend. Although CMM screening rates in Italy are unknown, the hypothesis that overdiagnosis has been a factor in the rise of incidence is plausible because largescale dermatology practice has reached levels of sensitivity for early CMM comparable to those of specialised clinics. ${ }^{50}$ For this reason, we are planning a high-resolution study of incidence trends by Breslow tumour thickness. ${ }^{51}$ In any case, it clearly appears that the incidence decrease occurring in the most recent cohorts cannot be related to overdiagnosis, unless one assumes that dermatologic surveillance has specifically weakened in this subset of the population. Such a hypothesis is unrealistic.

In conclusion, the changes observed in the latest generations of Italians are most likely the earliest manifestations of a birth-cohortdependent decrease in the risk of CMM. This large study adds to previous anecdotal data indicating that the reversal of the long-term increase in incidence of CMM is extending to southern Europe. It appears that the primary prevention strategies in Italy have been effective in modifying sun exposure habits of the latest birth cohorts, and should be reinforced.

\section{The AIRTUM Working Group}

The membership of the AIRTUM Working Group included the following: Chiara Balducci (Romagna Cancer Registry, Romagna Cancer Institute, Istituto Scientifico Romagnolo per lo Studio e la Cura dei Tumori (IRST) IRCCS, Meldola, Forli); Lorenza Boschetti (Pavia Cancer Registry, Public Health Agency of Pavia, Pavia); Barbara Braghiroli (Epidemiology Unit, Azienda Unità Sanitaria Locale - IRCCS di Reggio Emilia, Via Amendola 2, 42122 Reggio Emilia); Angelita Brustolin (Unit of Epidemiology and Cancer Registry, Local Health Authority, Viterbo); Giuseppa Candela (Trapani and Agrigento Cancer Registry, Trapani); Maurizio Castelli (Registro Tumori della Valle d'Aosta, Aosta); Rossella Cavallo (Cancer Registry - ASL Salerno, Salerno); Claudia Cirilli (Modena Cancer Registry, Public Health Department, Local Health Authority, Modena); Annarita Citarella (Cancer Registry, Department of Prevention, Unit of Epidemiology, Local Health Authority, Benevento); Roberta Cogno (Liguria Cancer Registry, IRCCS Ospedale Policlinico San Martino, Genova); Francesco Cuccaro (Barletta, Andria, Trani Cancer Registry, BAT Province, Barletta); Anna D'Agostino (Lombardy Cancer RegistryVarese Province, Cancer Registry Unit, Department of Research, Fondazione IRCCS Istituto Nazionale Tumori, Milan); Angelo D'Argenzio (Registro Tumori ASL di Caserta, Caserta); Nicoletta Feola (Sassari Cancer Registry, Azienda Regionale per la Tutela della Salute - ATS, Sassari); Stefano Ferretti (Romagna Cancer Registry, section of Ferrara, 
Local Health Authority and University of Ferrara, Ferrara); Anna Rita Fiore (Veneto Tumour Registry, Azienda Zero, Padova); Ilaria Fontanili (Parma Cancer Registry, Medical Oncology Unit, University Hospital of Parma, Parma); Graziella Frasca (Cancer Registry, Provincial Health Authority (ASP), Ragusa); Rocco Galasso (Unit of Regional Cancer Registry, Clinical Epidemiology and Biostatistics, IRCCS-CROB Basilicata, Rionero in Vulture); Cinzia Gasparotti (Registry of Brescia Province, Epidemiology Unit, Brescia Health Protection Agency, Brescia); Linda Guarda (Mantova Cancer Registry, Epidemiology Unit, Agenzia di Tutela della Salute (ATS) della Val Padana, Mantova); Teresa Intrieri (Tuscany Cancer Registry, Clinical and Descriptive Epidemiology Unit, Institute for Cancer Research, Prevention and Clinical Network (ISPRO), Florence); Walter Mazzucco (Department of Health Promotion, Maternal and Infant Care, Internal Medicine and Medical Specialties (PROMISE), University of Palermo, Palermo); Fernando Palma (Foggia Province Cancer Registry, Statistics and Epidemiology Unit, Asl Foggia, Foggia); Chiara Primieri (Department of Experimental Medicine, University of Perugia, Perugia); Roberto Rizzello (Trento Province Cancer Registry, Unit of Clinical Epidemiology, Trento); Giuseppe Sampietro (Bergamo Cancer Registry, ATS Bergamo, Bergamo); Santo Scalzi (Registro Tumori dell'ASP di Catanzaro); Salvatore Sciacca (Catania, Messina and Enna Cancer Registry, Catania); Margherita Tanzarella (Registro Tumori di Taranto); Federica Toffolutti (Cancer Epidemiology Unit, Centro di Riferimento Oncologico di Aviano (CRO) IRCCS, Aviano); Romina Vincenzi (Registro Tumori ASP Reggio Calabria); Maria Francesca Vitale (Napoli 3 Sud Cancer Registry, Napoli); Fabio Vittadello (South Tyrol Tumour Registry, Bolzano); and Roberto Zanetti (Piedmont Cancer Registry, A.O.U. Città della Salute e della Scienza di Torino, Turin, Italy).

\section{CONFLICT OF INTEREST}

The authors have no conflicts of interests to declare.

\section{ETHICS STATEMENT}

The study was approved by the Ethics Committee at the Romagna Cancer Institute (ID: IRST100.37).

\section{DATA AVAILABILITY STATEMENT}

Research data are available from the corresponding author upon reasonable request.

\section{ORCID}

Lauro Bucchi (D) https://orcid.org/0000-0002-6771-8119 Silvia Mancini (D) https://orcid.org/0000-0003-0673-3347 Emanuele Crocetti (D) https://orcid.org/0000-0002-4649-2034 Luigino Dal Maso (D) https://orcid.org/0000-0001-6163-200X Flavia Baldacchini (D) https://orcid.org/0000-0002-0557-798X Rosa Vattiato (iD https://orcid.org/0000-0002-3283-7572 Orietta Giuliani iD https://orcid.org/0000-0002-1843-3475 Alessandra Ravaioli (D) https://orcid.org/0000-0002-8778-6397 Giuliano Carrozzi (D) https://orcid.org/0000-0002-4405-6896 Stefano Ferretti (D) https://orcid.org/0000-0001-5241-2101 Rosa Angela Filiberti (D) https://orcid.org/0000-0002-9754-5394
Alessio Gili (D) https://orcid.org/0000-0002-4359-5193

Chiara Panato (D) https://orcid.org/0000-0002-3163-188X

Silvano Piffer (D) https://orcid.org/0000-0002-8316-3117

Massimo Rugge (D) https://orcid.org/0000-0002-0679-0563

Giovanna Tagliabue (iD https://orcid.org/0000-0001-8165-5524

Rosario Tumino (iD https://orcid.org/0000-0003-2666-414X

Ignazio Stanganelli (iD https://orcid.org/0000-0001-6575-8785

Fabio Falcini (D) https://orcid.org/0000-0002-0864-6266

\section{REFERENCES}

1. Bulliard JL, Cox B, Semenciw R. Trends by anatomic site in the incidence of cutaneous malignant melanoma in Canada, 1969-93. Cancer Causes Control. 1999;10:407-416.

2. Bulliard JL, Cox B. Cutaneous malignant melanoma in New Zealand: trends by anatomical site, 1969-1993. Int J Epidemiol. 2000;29: 416-423.

3. Stang A, Stang K, Stegmaier C, et al. Skin melanoma in Saarland: incidence, survival and mortality 1970-1996. Eur J Cancer Prev. 2001;10: 407-415.

4. MacKie RM, Bray CA, Hole DJ, et al. Incidence of and survival from malignant melanoma in Scotland: an epidemiological study. Lancet. 2002;360(9333):587-591.

5. Månsson-Brahme $\mathrm{E}$, Johansson $\mathrm{H}$, Larsson $\mathrm{O}$, et al. Trends in incidence of cutaneous malignant melanoma in a Swedish population 1976-1994. Acta Oncol. 2002;41:138-146.

6. de Vries E, Bray FI, Coebergh JW, et al. Changing epidemiology of malignant cutaneous melanoma in Europe 1953-1997: rising trends in incidence and mortality but recent stabilizations in western Europe and decreases in Scandinavia. Int J Cancer. 2003;107:119-126.

7. Montella A, Gavin A, Middleton R, et al. Cutaneous melanoma mortality starting to change: a study of trends in Northern Ireland. Eur J Cancer. 2009;45:2360-2366.

8. Holterhues $\mathrm{C}$, de Vries $\mathrm{E}$, Louwman $\mathrm{MW}$, et al. Incidence and trends of cutaneous malignancies in The Netherlands, 1989-2005. J Invest Dermatol. 2010;130:1807-1812.

9. Tryggvadóttir L, Gislum M, Hakulinen T, et al. Trends in the survival of patients diagnosed with malignant melanoma of the skin in the Nordic countries 1964-2003 followed up to the end of 2006. Acta Oncol. 2010;49:665-672.

10. Erdmann F, Lortet-Tieulent J, Schüz J, et al. International trends in the incidence of malignant melanoma 1953-2008: are recent generations at higher or lower risk? Int J Cancer. 2013;132:385-400.

11. Marrett LD, Nguyen HL, Armstrong BK. Trends in the incidence of cutaneous malignant melanoma in New South Wales, 1983-1996. Int J Cancer. 2001;92:457-462.

12. Hall HI, Miller DR, Rogers JD, et al. Update on the incidence and mortality from melanoma in the United States. J Am Acad Dermatol. 1999; 40:35-42.

13. Coory M, Baade P, Aitken J, et al. Trends for in situ and invasive melanoma in Queensland, Australia, 1982-2002. Cancer Causes Control. 2006;17:21-27.

14. Whiteman DC, Bray CA, Siskind V, et al. Changes in the incidence of cutaneous melanoma in the west of Scotland and Queensland, Australia: hope for health promotion? Eur J Cancer Prev. 2008;17: 243-250.

15. MacKie RM, Hauschild A, Eggermont AM. Epidemiology of invasive cutaneous melanoma. Ann Oncol. 2009;20(Suppl 6):vi1-vi7.

16. Giles GG, Armstrong BK, Burton RC, et al. Has mortality from melanoma stopped rising in Australia? Analysis of trends between 1931 and 1994. BMJ. 1996;312:1121-1125.

17. Aitken JF, Youlden DR, Baade PD, et al. Generational shift in melanoma incidence and mortality in Queensland, Australia, 1995-2014. Int J Cancer. 2018;142:1528-1535. 
18. Paulson KG, Gupta D, Kim TS, et al. Age-specific incidence of melanoma in the United States. JAMA Dermatol. 2020;156:57-64.

19. Thrift AP, Gudenkauf FJ. Melanoma incidence among non-Hispanic whites in all 50 United States from 2001 through 2015. J Natl Cancer Inst. 2020;112:533-539.

20. Autier P, Koechlin A, Boniol M. The forthcoming inexorable decline of cutaneous melanoma mortality in light-skinned populations. Eur $J$ Cancer. 2015;51:869-878.

21. Crocetti E, Carli P, Miccinesi G. Melanoma incidence in Central Italy will go on increasing also in the near future: a registry-based, ageperiod-cohort analysis. Eur J Cancer Prev. 2007;16:50-54.

22. Bianconi F, Crocetti E, Grisci C, et al. What has changed in the epidemiology of skin melanoma in Central Italy during the past 20years? Melanoma Res. 2020;30:396-401.

23. Buzzoni C, Crocetti E, Guzzinati S, et al. Cancer incidence and mortality trends from 2003 to 2014 in Italy. Tumori. 2019;105:121-137.

24. Ribes J, Clèries $R$, Buxó $M$, et al. Predictions of cancer incidence and mortality in Catalonia to 2015 by means of Bayesian models. Med Clin (Barc). 2008;131(Suppl 1):32-41.

25. Marcos-Gragera R, Vilar-Coromina N, Galceran J, et al. Rising trends in incidence of cutaneous malignant melanoma and their future projections in Catalonia, Spain: increasing impact or future epidemic? J Eur Acad Dermatol Venereol. 2010;24:1083-1088.

26. Arnold M, Holterhues $C$, Hollestein LM, et al. Trends in incidence and predictions of cutaneous melanoma across Europe up to 2015. J Eur Acad Dermatol Venereol. 2014;28:1170-1178.

27. Barbaric J, Sekerija M, Agius D, et al. Disparities in melanoma incidence and mortality in South-Eastern Europe: increasing incidence and divergent mortality patterns. Is progress around the corner? Eur $J$ Cancer. 2016;55:47-55.

28. Puig S, Marcoval J, Paradelo C, et al. Melanoma incidence increases in the elderly of Catalonia but not in the younger population: effect of prevention or consequence of immigration? Acta Derm Venereol. 2015;95:422-426.

29. Mancini S, Crocetti E, Bucchi L, et al. Time trends and age-periodcohort analysis of cutaneous malignant melanoma incidence rates in the Romagna region (northern Italy), 1986-2014. Melanoma Res. 2020;30:198-205.

30. Bray F, Colombet M, Mery L, et al. Cancer Incidence in Five Continents, Vol. XI (Electronic Version). Lyon: International Agency for Research on Cancer; 2017 Available at: http://ci5.iarc.fr (accessed June 13, 2020).

31. Associazione Italiana Registri Tumori. I tumori in Italia: trend 20032014. Available at: https://www.registri-tumori.it/cms/sites/default/ files/pubblicazioni/MONOGRAFIA_TREND_2003_2014.pdf. (Accessed 13 June 2020).

32. World Health Organization. International Statistical Classification of Diseases and Related Health Problems, 10th Revision. Vol 1. 5th ed. Geneva: World Health Organization; 2016:181.

33. Clayton D, Schifflers E. Models for temporal variation in cancer rates. I: age-period and age-cohort models. Stat Med. 1987;6:449-467.

34. Clayton D, Schifflers E. Models for temporal variation in cancer rates. II: age-period-cohort models. Stat Med. 1987;6:469-481.

35. Holford TR. Understanding the effects of age, period, and cohort on incidence and mortality rates. Annu Rev Public Health. 1991;12: 425-457.

36. Akaike $\mathrm{H}$. Information theory and an extension of the maximum likelihood principle. In: Petrov B, Csáki F, eds. Second International Symposium on Information Theory. Budapest: Akadémiai Kiadó; 1973: 267-281.
37. Rutherford MJ, Lambert PC, Thompson JR. Age-period-cohort modeling. Stata J. 2010;10:606-627.

38. Cayuela A, Rodríguez-Domínguez S, Vigil E, et al. Effect of age, birth cohort and period of death on skin melanoma mortality in Spain, 1975 through 2004. Int J Cancer. 2008;122:905-908.

39. Gutiérrez-González E, López-Abente G, Aragonés N, et al. Trends in mortality from cutaneous malignant melanoma in Spain (1982-2016): sex-specific age-cohort-period effects. J Eur Acad Dermatol Venereol. 2019;33:1522-1528.

40. Walkosz BJ, Buller D, Buller M, et al. Sun safe workplaces: effect of an occupational skin cancer prevention program on employee sun safety practices. J Occup Environ Med. 2018;60:990-997.

41. Istituto Nazionale di Statistica. I tempi della vita quotidiana. Available at: https://www.istat.it/it/files//2019/05/ebook-I-tempi-della-vitaquotidiana.pdf. (Accessed 13 June 2020).

42. Roberts WE. Skin type classification systems old and new. Dermatol Clin. 2009;27:529-533.

43. Whiteman DC, Green AC, Olsen CM. The growing burden of invasive melanoma: projections of incidence rates and numbers of new cases in six susceptible populations through 2031. J Invest Dermatol. 2016; 136:1161-1171.

44. Ascierto PA, AIRTUM Working Group. Melanoma. In: Associazione Italiana di Oncologia Medica, Associazione Italiana Registri Tumori, ed. I numeri del cancro in Italia: 2018. Brescia: Intermedia Editore; 2018:131-141.

45. Geller AC, Clapp RW, Sober AJ, et al. Melanoma epidemic: an analysis of six decades of data from the Connecticut tumor registry. J Clin Oncol. 2013;31:4172-4178.

46. Boscolo-Rizzo P, Zorzi M, Del Mistro A, et al. The evolution of the epidemiological landscape of head and neck cancer in Italy: is there evidence for an increase in the incidence of potentially HPV-related carcinomas? PLoS One. 2018;13:e0192621.

47. Dal Maso L, Lise $M$, Zambon $P$, et al. Incidence of thyroid cancer in Italy, 1991-2005: time trends and age-period-cohort effects. Ann Oncol. 2011;22:957-963.

48. Mancini S, Bucchi L, Baldacchini $F$, et al. Incidence trends of vulvar squamous cell carcinoma in Italy from 1990 to 2015. Gynecol Oncol. 2020;157:656-663.

49. Maso D, Panato C, Franceschi S, et al. The impact of overdiagnosis on thyroid cancer epidemic in Italy, 1998-2012. Eur J Cancer. 2018;94:6-15.

50. Argenziano G, Moscarella E, Annetta A, et al. Melanoma detection in Italian pigmented lesion clinics. G Ital Dermatol Venereol. 2014;149: 161-166.

51. Sacchetto L, Zanetti R, Comber $\mathrm{H}$, et al. Trends in incidence of thick, thin and in situ melanoma in Europe. Eur J Cancer. 2018;92:108-118.

\section{SUPPORTING INFORMATION}

Additional supporting information may be found online in the Supporting Information section at the end of this article.

How to cite this article: Bucchi L, Mancini S, Crocetti E, et al. Mid-term trends and recent birth-cohort-dependent changes in incidence rates of cutaneous malignant melanoma in Italy. Int. J. Cancer. 2020;1-10. https://doi.org/10.1002/ijc.33259 